\title{
Absentismo escolar en Educación Primaria. Medidas de prevención y atención educativa
}

\author{
Bejarano Prats, Purificación \\ Centro de Magisterio Sagrado Corazón, Córdoba, España \\ pbejarano@uco.es
}

\section{Resumen}

El absentismo escolar es una problemática que se origina por variedad de causas y situaciones, puede derivarse de factores de índole personal o en relación con el entorno (familiar, escolar y/o social). Las faltas de asistencia continuadas y sin justificación en la enseñanza obligatoria, tienen consecuencias negativas en el proceso educativo del alumnado. Este fenómeno se relaciona con situaciones de fracaso escolar y abandono temprano. Además, las dificultades derivadas de absentismo escolar incrementan en el caso de estudiantes que se sitúan en zonas socio- económicamente desfavorecidas, acrecentando su riesgo de exclusión social.

Este artículo analiza el absentismo escolar en la etapa de Educación Primaria y en la comunidad autónoma de Andalucía, profundizando en medidas educativas establecidas para su prevención y control. Se incide en la importancia del desarrollo de estrategias preventivas y de la necesidad de una acción integral, que requiere la intervención adecuada de todos los sectores de la comunidad educativa y otros profesionales de organismos o entidades competentes.

\section{Abstract}

School absenteeism is a problem caused by different reasons and situations and can arise from personal factors or be related to family, school and/or social environment. The prevalence of unexcused absences in compulsory education, have negative consequences on children's educational process. It is related to educational failure and early school leaving. Furthermore, the problems come from school absenteeism improve in case of students from socio-economic disadvantage areas, increasing the social exclusion risk. This article analyses school absenteeism in Primary Education stage and in the Autonomous Community of Andalucía, deepening on educational measures established to its prevention and control. It is insisted on the importance of the development of preventive strategies and the necessity of an integral action, which needs the appropriate intervention of all the sectors of the educational community and other professionals of relevant agencies and entities.

Palabras clave: absentismo, educación compensatoria, enseñanza primaria, estrategias educativas.

Keywords: absenteeism, compensatory education, primary education, educational strategies.

\section{INTRODUCCIÓN}

El compromiso educativo para la eliminación del absentismo escolar se hace evidente y resulta necesario establecer medidas desde diferentes sectores. Se deben ofrecer respuestas desde «una mirada atenta y profunda, una mirada multidimensional» (García, 2013, p. 11) pues se deben examinar las circunstancias personales y sociales que conllevan a estasituación.

De acuerdo con Moyano, Ramírez, Martos y Anguita (2017) «el absentismo escolar es un factor de riesgo para la exclusión y la desigualdad, constituyendo un reto para los sistemas educativos europeos» (p. 65). Generalmente, alumnado procedente de contextos socio-económicamente desfavorecidos manifiestan irregularidades en la asistencia a los centros, desencadenando situaciones de absentismo o abandono prematuro. 
En España, el Plan para la Reducción del Abandono Educativo Temprano expresa la importancia de «conseguir que las personas sigan un proceso educativo con éxito y eliminar el absentismo escolar» (Ministerio de Educación, Cultura y Deporte, 2014, p. 9). De hecho, este es un reto planteado en la normativa que regula el sistema escolar español, concretamente en la exposición de motivos de la Ley Orgánica de Mejora de la Calidad Educativa (LOMCE, 2013) se expresa la influencia de «las elevadas tasas de abandono temprano de la educación» (p. 97861) como un aspecto que intercede de manera negativa en el progreso de la calidad educativa, siendo esta «una de las debilidades del sistema» (p. 97861) y uno de «los principales objetivos que persigue la reforma» (p. 97862).

Si bien, ambos fenómenos, abandono educativo temprano y absentismo escolar están relacionados y su prevención y control suponen un reto en el sistema escolar, resulta conveniente diferenciarlos, aunque como índica García (2013) «no siempre es fácil discernir, a lo largo del curso escolar, cuándo se pasa de una situación de absentismo al abandono escolar»(p. 28). Por tanto, brevemente se puede indicar que el primero se refiere a ausencias continuadas del alumnado durante la enseñanza obligatoria sin causas justificadas (Decreto 167/2003, 2003), mientras que el segundo implica la no asistencia a la escuela de manera definitiva en edad reglamentaria (García,2013).

Pues bien, en los últimos años se han ido planteando diversas medidas para paliar estas problemáticas, de tal forma se han ido proponiendo actuaciones en distintos niveles, «planes más globales, autonómicos y municipales» (González, 2014, p. 6) en los que se han basado centros escolares para plantear sus propuestas. El presente artículo se centra en el absentismo escolar en Educación Primaria y en Andalucía. Concretamente en esta Comunidad Autónoma también se establecen disposiciones legislativas para conseguir la disminución del absentismo, aspecto propuesto en el Plan de Éxito Educativo planteado para los cursos 2016 a 2020 (Consejería de Educación de la Junta de Andalucía,2016).

Es una problemática que requiere medidas educativas eficaces, suele concentrarse en determinadas zonas y centros escolares, concretamente en el año 2017 los medios de comunicación hacen eco de que la Policía ha intervenido en 428 casos de absentismo escolar de alumnado matriculado en la enseñanza obligatoria (Europa Press, 2018). Si bien se plantean medidas a nivel institucional, resulta difícil cuantificar el absentismo escolar, indica García (2013) que «la tasa de absentismo no es un indicador recogido por las estadísticas regulares de educación (...) la ausencia de información estadística contribuye a la invisibilidad del absentismo» (p. 29). Por tanto, se pretende promover la necesidad de identificar las situaciones de absentismo en Andalucía, reconocer causas que las producen, el protocolo de intervención y medidas educativas que puedan llevarse a cabo, tanto para la prevención como para el control de dicho fenómeno.

\section{CAUSAS DE ABSENTISMO ESCOLAR}

La adecuada actuación para prevenir y controlar el absentismo escolar requiere conocer las causas que originan esta situación. Sucesivamente se especifican razones de carácter general, no obstante para adoptar medidas precisas a cada situación se deben identificar en cada caso de manera particular.

Las causas son muy diversas, pueden ser múltiples los factores que inciden y de diversa índole, derivados del contexto familiar, escolar y/o social. La siguiente tabla puede ilustrar dicha clasificación, establecida en el Acuerdo de 25 de noviembre de 2003 (2003). 


\begin{tabular}{|c|l|}
\hline \multirow{4}{*}{ Ámbitos } & \multicolumn{1}{|c|}{ Indicadores } \\
\hline \multirow{5}{*}{ Familiar } & $\begin{array}{l}\text { Despreocupación de los padres por la asistencia de los hijos a la escuela ya que no la } \\
\text { consideran importante. }\end{array}$ \\
\cline { 2 - 3 } & La dedicación de los menores a tareas laborales consentidas por las familias. \\
\cline { 2 - 3 } & $\begin{array}{l}\text { La necesidad de que chicas jóvenes en edad deescolaridad obligatoria se tengan que en- } \\
\text { cargar del cuidado de los hermanos por la actividad laboral de los padres. }\end{array}$ \\
\cline { 2 - 3 } & Otras problemáticas derivadas con el alcoholismo, drogodependencias, etc. \\
\hline \multirow{5}{*}{ Social } & Condiciones desfavorables del barrio o de la zona. \\
\cline { 2 - 3 } & Incidencia negativa en el alumno del grupo deiguales. \\
\cline { 2 - 3 } & Problemáticas en el menor referidas a distintas adicciones. \\
\hline \multirow{5}{*}{ Escolar } & Falta de adaptación de la escuela a las características delcalumnado. \\
\cline { 2 - 3 } & Inadaptación del estudiante a la institución escolar. \\
\cline { 2 - 3 } & Rechazo a la escuela. \\
\cline { 2 - 3 } & Desmotivación del alumnado a las tareas escolares. \\
\hline
\end{tabular}

Nota. Elaboración propia, según Acuerdo de 25 de noviembre de 2003.

Los motivos por los que se produce el absentismo escolar "expresan una forma de desigualdad educativa» (García, 2013, p. 33) y esta problemática incrementa dicha situación, por lo que resulta necesaria su prevención y eliminación. Por tanto, se deben establecer medidas con rapidez y eficacia en función de las causas que lo provocan. Sucesivamente se especifica el protocolo de intervención establecido en Andalucía.

\section{PROTOCOLO DE INTERVENCIÓN}

Una situación de absentismo escolar durante la etapa de Educación Primaria queda regulada en Andalucía, "cuando las faltas de asistencia sin justificar al cabo de un mes sean de cinco días lectivos» (Orden de 19 de septiembre de 2005, 2005, p. 7). Dicha orden recoge en su artículo 8, el procedimiento aseguir:

- Se realizará control de la asistencia diario por los tutores y en el momento en que se detecte una situación de absentismo se encargarán de mantener una entrevista con las familias o representantes legales de los estudiantes para identificar las posibles causas de las faltas de asistencia injustificadas del alumnado y establecer un compromiso para solventar la situación.

Conviene matizar, según añade el artículo 10 que es competencia del docente de Apoyo a la Compensación Educativa que forma parte del Equipo de Orientación Educativa (EOE) «el tratamiento y análisis de los datos proporcionados por los centros así como el impulso y coordinación de las actuaciones que se desarrollen en este campo en los centros docentes de su zona de actuación» (Orden de 19 de septiembre de 2005, 2005, p. 7).

- Si se resuelve la situación finaliza el proceso, en caso de no obtener respuesta por parte de la familia o se incumple lo acordado, el tutor informará a Jefatura o a la Dirección del Centro para que expongan por escrito la situación y las responsabilidades que incumplen. También se comunica a los Servicios Sociales Comunitarios o a los Equipos Técnicos de Absentismo Escolar (ETAE)', que se encargarán de establecer las medidas pertinentes.

\footnotetext{
${ }^{1}$ ETAE: constituido en cada localidad o municipio integrado por los profesionales que más directamente intervienen en esta problemática (Equipo de Orientación Educativa y Departamento de Orientación, responsables de los Centros Educativos, Servicios Sociales Municipales o Comunitarios, Policía Local) y que concretará en cada localidad las actuaciones priorizadas por la Comisión Municipal (Orden de 19 de septiembre de 2005, 2005, cláusula sexta1).
} 
- Cuando no funcionan las intervenciones establecidas por los Servicios Sociales o los ETAE se deriva el caso a la Comisión y/o Subcomisión Municipal de Absentismo Escolar².

- En las situaciones en que «las diferentes medidas adoptadas no den los resultados satisfactorios y se aprecie una posible situación de desprotección en los menores, se trasladará la información a la Fiscalía de Menores y al Servicio de Protección de Menores» (Orden de 19 de septiembre de 2005, 2005, p. 7) según añade el artículo 9 de la citada normativa.

Identificado el procedimiento que se debe seguir, según establece la normativa vigente en Andalucía en situaciones de absentismo escolar, sucesivamente se esbozan líneas prioritarias de actuación en la prevención y control desde diferentes ámbitos y atendiendo a unos principios básicos de actuación.

\section{MEDIDAS EDUCATIVAS DE PREVENCIÓN Y CONTROL}

Establecer medidas para la prevención, anticiparse a circunstancias sociales, escolares y familiares que originan absentismo escolar en los estudiantes en edad de la enseñanza obligatoria es fundamental para evitar esta problemática y una mayor situación de desigualdad. Asimismo, las actuaciones propuestas deben ser integrales, es decir, plantearse desde diferentes ámbitos, no solo el educativo, de hecho resulta necesaria la coordinación interadministrativa (a nivel autonómico, provincial y local), pues se debe intervenir de manera focalizada, poner mayor énfasis en determinadas zonas de transformación social e implicar a las familias, pues estas son fundamentales para evitar el absentismo escolar (Acuerdo de 25 de noviembre de 2003, 2003).

A nivel legislativo se especifican actuaciones que deben desarrollarse para la prevención y control del absentismo escolar. En este sentido, en la comunidad autónoma de Andalucía se indica en la Ley 19/1999, de 18 de noviembre, de Solidaridad en la Educación el desarrollo de programas de compensación educativa, entre otros aspectos para luchar contra el absentismo. Se especifica en el Decreto 167/2003, de 17 de junio, la ordenación de la atención educativa a los alumnos y alumnas con necesidades educativas especiales asociadas a condiciones sociales desfavorecidas, concretamente el Título III se refiere a los programas de lucha contra el absentismo escolar.

A propuesta de la Consejería de Educación y Ciencia se aprueba el Plan Integral para la Prevención, Seguimiento y Control del Absentismo Escolar, así se establece el Acuerdo de 25 de noviembre de 2003 donde se regulan áreas de intervención, con sus correspondientes objetivos generales, específicos, medidas a establecer y los órganos responsables en cada una de ellas (véase el apartado cuarto del Acuerdo de 25 de noviembre de 2003).

Posteriormente, la Orden de 19 de septiembre de 2005 regula determinados aspectos del acuerdo anteriormente citado, concretando actuaciones del Plan Integral para la Prevención, Seguimiento y Control del Absentismo Escolar. Se crea una Comisión Interdepartamental de Absentismo Escolar con la finalidad de favorecer la coordinación.

Así, se deben establecer medidas, como se indicó anteriormente, en función de las causas que generan el absentismo escolar, por ello se especifican las diferentes áreas de actuación. En la siguiente tabla pueden observarse posibles actuaciones para tenerse en cuenta, clasificadas según las circunstancias de carácter familiar, social y escolar.

\footnotetext{
${ }^{2}$ Comisión Municipal de Absentismo Escolar: estará formada por el alcalde o persona en quien delegue, concejales delegados (del área de Educación, de Servicios Sociales, de Seguridad Ciudadana), representante de la Delegación Provincial de Educación, representante de la Delegación Provincial de Igualdad y Bienestar Social, responsable de los Servicios Sociales de la localidad o municipio, director de los centros de Educación Primaria y Secundaria en los que se lleven a cabo actuaciones contra el absentismo escolar, representante de la Policía Local, representante de las Asociaciones de Padres y Madres de centros de Educación Primaria y Secundaria en los que se lleven a cabo actuaciones para solucionar el absentismo escolar; representante, en su caso, de las asociaciones no gubernamentales que desarrollen actuaciones encaminados a eliminar del absentismo escolar en el municipio (Orden de 19 de septiembre de 2005, 2005, cláusula quinta 1).
} 
Tabla 2. Medidas de actuación en el control del absentismo escolar, en función de las causas que lo originan.

\begin{tabular}{|c|c|}
\hline Ámbitos & Medidas \\
\hline \multirow{4}{*}{ Familiar } & Concienciar y motivar a las familias por la asistencia de los hijos a la escuela. \\
\hline & Favorecer la participación de las familias con el tutor y en actividades escolares. \\
\hline & $\begin{array}{l}\text { Trabajo coordinado con la familia (desde el centro educativo,persona encargada del EOE, } \\
\text { ETAE, Servicios Sociales comunitarios) para intentar solventar la situación problemática. }\end{array}$ \\
\hline & $\begin{array}{l}\text { Favorecer la escolarización continuada en los centros escolares de origen en el alumnado } \\
\text { cuyos padres se dediquen a tareas agrícolas de temporada o profesiones itinerantes. }\end{array}$ \\
\hline \multirow{3}{*}{ Social } & Modificar la relación del estudiante con el entorno social en el que se encuentra. \\
\hline & $\begin{array}{l}\text { Crear actividades lúdicas y hacer posible la ocupación del alumnado en el tiempo de ocio en } \\
\text { actividades educativas. }\end{array}$ \\
\hline & Mejorar la comunicación entre servicios sociales municipales y los centros docentes. \\
\hline \multirow{3}{*}{ Escolar } & Establecer estrategias en los centros escolares que favorezcan la adaptación del alumnado. \\
\hline & Ofrecer una enseñanza atractiva y motivadora. \\
\hline & $\begin{array}{l}\text { Favorecer la implicación y trabajo coordinado entre equipo directivo, equipos docentes, EOE } \\
\text { y trabajadores sociales o educadores sociales. }\end{array}$ \\
\hline
\end{tabular}

Nota. Elaboración propia, según Acuerdo de 25 de noviembre de 2003 (2003).

Si bien, algunas de estas medidas se pueden utilizar tanto para la intervención en situaciones de absentismo, como para la prevención de este fenómeno (véase el artículo 4 de la Orden de 19 de septiembre de 2005), se destaca la necesidad de favorecer la relación con las familias, ofrecer los recursos necesarios (como el comedor escolar), desarrollar una adecuada formación docente y atender desde el ámbito escolar a las necesidades del alumnado favoreciendo su educación integral. También, se desea realizar especial énfasis en la medida que se plantea sobre la necesidad de diseñar y aplicar planes para «el fomento de las expectativas de éxito escolar, el trabajo en grupo, el desarrollo de la creatividad y el incremento de la autoestima» (Acuerdo de 25 de noviembre de 2003, 2003, p. 25627). Por último, como se establece en dicho Acuerdo, los centros escolares «deben poner todas las medidas de carácter organizativo, pedagógico y curricular que sean necesarias para ofrecer una enseñanza atractiva y motivante» (p. 25624), en esta línea, se promueve la necesidad de establecer en los centros educativos estrategias que favorezca la motivación, tanto del alumnado, como de los docentes y las familias.

\section{DISCUSIÓN Y CONCLUSIONES}

Analizadas las causas y actuaciones para la prevención y control del absentismo escolar, se impulsa el desarrollo de tres áreas de trabajo que se consideran importantes para focalizar la intervención realizada y mejorar la labor que en estos ámbitos se plantee:

El primero se refiere a la importancia de reconocer las causas que pueden generar o ya están ocasionando una situación de absentismo escolar. Establecer medidas adecuadas, tanto para la prevención como para el control de este fenómeno, requiere una atención personalizada a cada caso. Es importante conocer al alumnado y su familia, las características del centro y del entorno, en definitiva se trata de observar y analizar indicadores que pueden ocasionar en cada situación particular absentismo. Y en este sentido se debe incidir no solo en el control, sino también en suprevención. 
En segundo lugar se considera fundamental el desarrollo de la autoestima, la creatividad y la motivación en el alumnado, familias y docentes. Desarrollar estrategias que favorezcan estos aspectos en el ámbito escolar se considera fundamental para la prevención del absentismo escolar. Para ello, los docentes deben tener una adecuada formación en este sentido, su actitud hacia el grupo y su manera de proceder incidirán en la autoestima, creatividad y motivación el alumnado y las familias, sin duda, se considera decisivo mejorarlos en la persona.

En tercer lugar se requieren actuaciones de una manera integral y con la colaboración de distintos profesionales. Establecer cauces para una adecuada coordinación entre los profesionales del centro escolar e intentar fomentar una comunicación fluida con las familias, favoreciendo la relación entre toda la comunidad educativa, así como la importancia de consolidar redes con profesionales de otras instituciones y entidades que permitan una intervención efectiva yadecuada.

\section{REFERENCIAS BIBLIOGRÁFICAS}

Acuerdo de 25 de noviembre de 2003, del Consejo de Gobierno, por el que se aprueba el Plan Integral para la Prevención, Seguimiento y Control del Absentismo Escolar. BOJA n. ${ }^{\circ} 235$.

Consejería de Educación de la Junta de Andalucía (2016). Plan de Éxito Educativo 2016-2020. Recuperado de http://www. juntadeandalucia.es/export/drupaljda/Plan_de_Exito_Educativo.pdf.

Decreto 167/2003, de 17 de junio, por el que se establece la ordenación de la atención educativa a los alumnos y alumnas con necesidades educativas especiales asociadas a condiciones sociales desfavorecidas, BOJA n. ${ }^{0} 118$ (2003).

Europa Press (2018). La Policía Adscrita intervino en 428 casos de absentismo escolar en Andalucía durante 2017. Publicado 27 de febrero de 2018. Recuperado de http://www.europapress.es/esandalucia/sevilla/noticia-policia-adscrita-intervino-428-casos-absentismo-escolar-andalucia-2017-20180227113308.html.

García, M. (2013). Absentismo y abandono escolar. Madrid: Editorial Síntesis.

González, M. T. (2014). Absentismo escolar: posibles respuestas desde el centro educativo. Revista Iberoamericana sobre Calidad, Eficacia y Cambio en Educación, 12(2), 5-27. Recuperado de http://www.redalyc.org/html/551/55130462001/

Ley 19/1999, de 18 de noviembre, de Solidaridad en la Educación. BOJA n. ${ }^{\circ} 140$ (1999).

Ley Orgánica 2/2007, de 19 de marzo, de reforma del de Estatuto de Autonomía para Andalucía, BOE n. ${ }^{\circ} 68$ (2007).

Ley Orgánica 8/2013, de 9 de diciembre, para la mejora de la calidad educativa, BOE $n^{\circ} 295$ (2013). Ministerio de Educación, Cultura y Deporte (2014). Plan para la Reducción del Abandono Educativo.

Temprano. Educación y formación. Periodo 2014-2020. Recuperado de https://www.mecd.gob.es/dms/mecd/educacion-mecd/areas-educacion/sistema-educativo/estudios-sistemas-educativos/espanol/especificos/estrategia-competencias- ocde/documentacion/Plan-para-la-reducci-n-del-abandono-eductivo-temprano.pdf.

Moyano, M., Ramírez, A., Martos, M. ${ }^{a}$ D. y Anguita, V. (2017). El absentismo escolar en Andalucía (España): balance y propuestas de futuro en el marco de la Unión Europea. Recuperado de http://www.redalyc.org/pdf/310/31054991004.pdf.

Orden de 19 de septiembre de 2005, por la que se desarrollan determinados aspectos del Plan Integral para la Prevención, Seguimiento y Control del Absentismo Escolar. BOJA n. ${ }^{\circ} 202$ (2005). 